\title{
Aspecto clínico de los casos de meningitis meningocóccica que se presentaron en Valparaíso de 31 de mayo al 12 de septiembre de 1941 en la sección niños del Hospital Deformes
}

\author{
Publicado en la Revista Chilena Pediatría de 1942
}

Autores: HÉCTOR PUMARINO, VÍCTOR GROSSI

Comentario: LUISA SCHONHAUT B. ${ }^{1}$

1. Profesor. Pediatra, Departamento de Pediatría. Facultad de Medicina Clínica Alemana - Universidad del Desarrollo.

Key words: Neisseria meningitidis, Epidemiology, Meningococus, Pediatric history.

Palabras clave: Neisseria meningitidis, Epidemia, Meningococo, Historia pediatría.

\section{Introducción}

En Chile, los primeros casos meningitis cerebro espinal epidémica fueron descritos en los años 20 por los Drs. Cuevas y posteriormente Casasbella. Durante las siguientes dos décadas el total de casos notificados fue un poco más de 501,2 "fuera de un pequeño brote, habido en 1936, en la isla Quiriquina y de unos cuantos casos que se presentaban casi todos los años y cuya filiación quedaba habitualmente en la penumbra, no los ha habido en cantidad digna de consideración" 3 .

No obstante, a mediados del año 1941 llegó una devastadora epidemia, que comenzó en el asilo de niños de Valparaíso, ciudad en que la tasa de ataque alcanzó 89,2/100 000 habitantes el año 1941 y 188,1/100 000 al año siguiente ${ }^{2}$. En un lapso inferior a 4 años se contabilizaron
9669 casos a lo largo del país, de los cuales la cuarta parte fue diagnosticada en la capital ${ }^{4}$ (Figura 1).

Según refiriese el Dr. Hernán Romero "En el estudio de la epidemia actual de meningococia, partimos de la hipótesis de que se trata de una enfermedad nueva en Chile"; probablemente ese sería el motivo de la falta de inmunidad en la población, que reunía además otros factores de riesgo, como desnutrición, hacinamiento y condiciones climáticas desfavorables ${ }^{2}$. A partir de entonces, la incidencia de enfermedad meningocócica fue descendiendo en forma sostenida hasta nuestros días.

Dada la contingencia actual frente a la epidemiología y profilaxis del meningococo, revisada en profundidad en el presente número de la revista ${ }^{5}$, nos pareció de gran interés rescatar las primeras publicaciones desde los archivos de hace casi 75 años. Si bien han pasado varias décadas y enormes progresos terapéuticos desde la publicación de los Drs. Pumarino y Grossi, los desafíos que nos plantea el meingococo se han mantenido vigentes ${ }^{6}$.

Trabajo recibido el 10 de noviembre de 2012, aceptado para publicación el 1 de diciembre de 2012.

Correspondencia a:

Luisa Schonhaut B.

E-mail: Ischonhaut@alemana.cl 


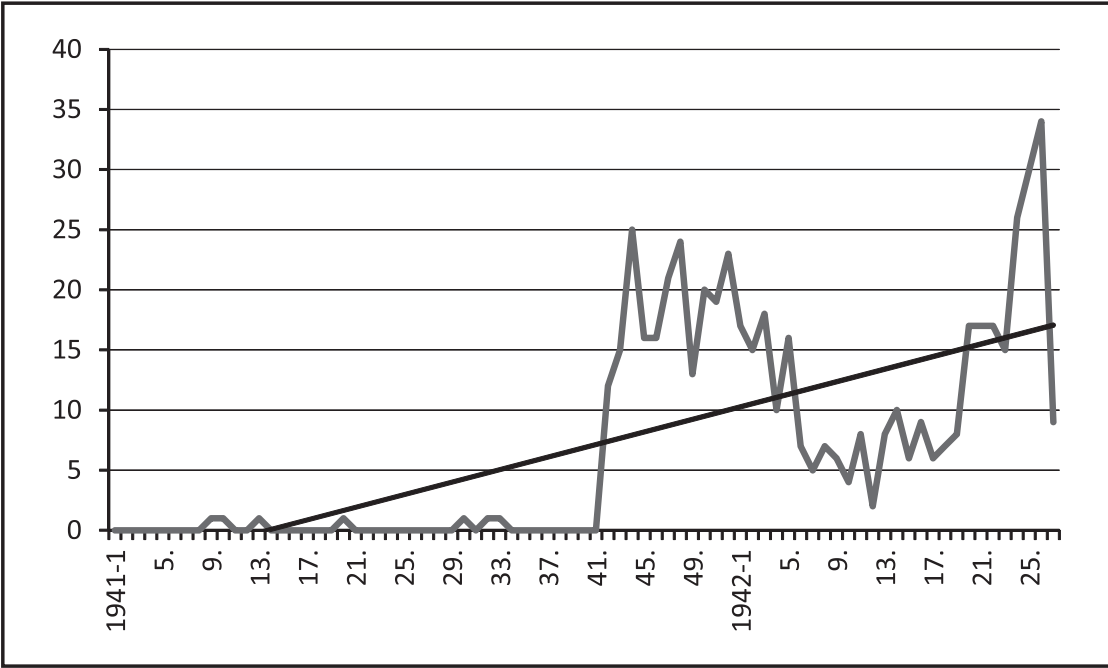

Figura 1. Casos de meningitis notificados entre los meses de enero 1941 y julio 1942 en la ciudad de Santiago (Análisis semanal). Adaptado de H Romero $1943^{3}$.

\section{Artículo}

Hemos creído como laborantes de servicios clínicos y sanitarios que era de interés para las corporaciones médicas y sanitarias, el estudio comparativo del grupo tan numeroso de una enfermedad muy rara entre nosotros y la experiencia terapéutica recogida en nuestro servicio.

Considerarnos nuestra experiencia aun muy escasa y procuraremos presentar los 40 casos observados en la forma más objetiva posible y aun cuando llegamos a algunas conclusiones, éstas no son aún definitivas.

\section{Historia}

Pocos trabajos clínicos encontrados en nuestra literatura sobre esta enfermedad hasta el año 1920, en que el Dr. Carlos Cuevas llama la atención sobre ella en la Sociedad Médica de Valparaíso, presentando el cerebro de un sujeto muerto de meningitis, que a pesar de no haberse hecho examen bacteriológico anatomo-patológicamente hacía sospechar lo fuera de meningitis cerebro espinal.

El año 1923 el Dr. Casasbella, de Santiago, presentó el primer caso comprobado bacteriológicamente por el Dr. Sepúlveda. Desde entonces hasta el año 1940, inclusive, se han presentado una cincuentena de casos más bien aislados en forma esporádica, sin alcanzar ja- más los caracteres y la extensión del actual brote que hemos tenido en Valparaíso.

Revisando los archivos del Hospital de Niños y de la sección niños del Hospital Deformes de Valparaíso de 10 años a esta parte, hemos encontrado 43 niños afectos de meningitis purulenta: 20 en el Hospital de Niños, cuyas edades fluctúan entre un mes seis días hasta los 11 años; y 23 del Hospital Deformes desde los 3 meses hasta los 12 años.

Tenemos así los siguientes datos:

\begin{tabular}{|c|c|c|c|c|}
\hline Año & $\begin{array}{l}\text { Nú- } \\
\text { mero }\end{array}$ & Edad & Sexo & $\begin{array}{l}\text { Estación } \\
\text { del año }\end{array}$ \\
\hline 1931 & 4 & $\begin{array}{c}1 \text { a } 3 \text { meses }= \\
3\end{array}$ & $\begin{array}{l}\text { Masculino } \\
27\end{array}$ & $\begin{array}{c}\text { Primavera }= \\
13\end{array}$ \\
\hline 1932 & 1 & $\begin{array}{c}3 \text { a } 6 \text { meses }= \\
4\end{array}$ & $\begin{array}{c}\text { Femenino } \\
16\end{array}$ & $\begin{array}{c}\text { Verano }= \\
9\end{array}$ \\
\hline 1933 & 1 & $\begin{array}{c}6 \text { a } 12 \text { meses }= \\
7\end{array}$ & & $\begin{array}{c}\text { Otoño }= \\
5\end{array}$ \\
\hline 1934 & 3 & $\begin{array}{c}1 \text { a } 2 \text { años }= \\
11\end{array}$ & & $\begin{array}{c}\text { Invierno }= \\
16\end{array}$ \\
\hline 1935 & 10 & $\begin{array}{c}2 \text { a } 6 \text { años }= \\
7\end{array}$ & & \\
\hline 1936 & 3 & $\begin{array}{c}6 \text { a } 12 \text { anos }= \\
11\end{array}$ & & \\
\hline 1937 & 5 & & & \\
\hline 1938 & 1 & & & \\
\hline 1939 & 3 & & & \\
\hline 1940 & 6 & & & \\
\hline $\begin{array}{l}1941 \\
\text { (hasta } \\
\text { mayo) }\end{array}$ & 6 & & & \\
\hline
\end{tabular}


De estos 43 casos, se pueden catalogar como meningitis meningocóccica por la sintomatología clínica y caracteres del liquido céfalo raquídeo (líquido purulento y diplococus Gram negativos al examen directo) 4 casos.

Diplococus catarralis 7 casos.

Bacilo de Fridlander 1 caso.

Neumococos 1 caso.

El 31 de Mayo del presente año, llegaba a la sección Pediatría del Hospital Deformes un niño de 3 años, asilado en la Casa de Huérfanos de la Providencia de Valparaíso, con un cuadro clínico que en un comienzo nos desorientó.

Había empezado su enfermedad el día anterior con vómitos, cefalea, dolor abdominal y temperatura.

Examinado el niño se le encontró en un estado angustioso, sensorio semi-obnubilado, hiperestesia cutánea, traspiración profusa y febril, rigidez de la nuca.

En un comienzo se pensó en un cuadro de intoxicación alimenticia y aún en la posibilidad de un abdomen agudo. Sin embargo, por ciertas características, se pensó en una meningitis aguda y se hizo una punción lumbar, dando salida a líquido turbio purulento a presión.

El resultado del examen al día siguiente nos puso sobre la pista. Teníamos entre nosotros un caso de meningitis cerebro espinal, que a pesar de no haberse hecho el cultivo, teníamos fundamento para pensar en ella (líquido purulento con Diplococus Gram negativo al examen directo).

Tres días después ingresaba otro niño del mismo asilo con un carácter análogo y el resultado del examen, el líquido y el cultivo positivo paira el meningococo nos daban razón para pensar que estábamos en presencia de casos de meningitis meningocóccica.

En pocos días teníamos 5 niños procedentes del asilo cuyas investigaciones dieron resultados positivos para el meningococo y al poco tiempo comenzaban a llegar de diferentes puntos de la ciudad, Viña del Mar y ciudades más alejadas, como Quilpué, Casablanca, Villa Alemana, etc., de nuevos enfermitos con cuadros meníngeos, hasta llegar en la actualidad a un total de 43; de estos son: 40 casos de meningitis meningocóccica; un caso de meningitis hiperaguda (fulminante, probablemente meningocóccica) 2 casos de meningitis purulenta o Bacilo de Pfeiffer.

De los 40 casos de meningitis meningocóccica tenemos 14 con cultivo positivo, las restantes, si bien es cierto, en algunos de ellos no se hizo cultivos y en otros fue negativo, hay méritos suficientes para pensar en la meningitis meningocóccica por los antecedentes, cuadro clínico, el aspecto y los caracteres del líquido y en casi todos ellos se encontró el Diplococus Gram negativo intra o extracelulares.

De las dos meningitis por bacilos del Pfeiffer, el resultado del cultivo fue positivo, una falleció al día siguiente en su casa por exigir la madre el alta, y la otra se encuentra buenas condiciones y lleva 20 días en el hospital.

A raíz de presentarse varios casos de meningitis en la Providencia, la Jefatura Provincial de Sanidad destacó allí personal especial para tomar medidas profilácticas. Una de estas medidas fue dar a 20 niños de 30 que habían estado en contacto con los enfermos, una dosis de sulfonilamida durante 14 días (períodos de incubación) a fin de protegerlos de la enfermedad.

De las 40 meningitis meningocóccica de que nos vamos a ocupar, sólo 4 fallecieron, lo que nos da un porcentaje de mortalidad de un $10 \%$. que consideramos muy satisfactorio.

Una característica interesante es dable constatar en casi todos los brotes de esta enfermedad, es el hecho de presentarse como casos aislados, apareciendo en puntos distintos unos de otros y de presentarse, en su inmensa mayoría, en hogares de gente modesta, en donde hay miseria y hacinamiento.

Se le da gran importancia al hacinamiento y el contagio se haría especialmente en los sitios cerrados, de preferencia en los dormitorios.

Según las últimas informaciones, Londres habría tenido un gran aumento de la morbilidad por meningitis meningocóccica y esta difusión se atribuyó al hacinamiento de los refugios anti aéreos. De aquí la importancia de contar con salas especiales donde pueda hacerse la atención y tratamiento de estos enfermos.

Leybour, analizando 5 brotes epidémicos en el Estado de Texas, llega a las siguientes conclusiones: los portadores de ropas esporá- 
dicas y saprofitos de meningococos no revisten importancia epidemiológica en los brotes; el portador peligroso es el de tipo intermitente crónico, pues puede trasmitir de cuando en cuando infecciones masivas.

Para que pueda triunfar un meningococo de mediana agresividad, precisa una disminución de la resistencia general del individuo y una infección masiva.

Las enfermedades trasmisibles deben dividirse en dos grupos: los de susceptibilidad universal y epidemiológica bien definida y los de susceptibilidad limitada y epidemiología oscura.

Entre los primeros tenemos la tifoidea, viruela, parotiditis y sarampión. Entre las segundas, meningitis meningocóccicas, poliomielitis, neumonía y fiebre ondulante.

\section{Cuadro Clínico}

En la gran mayoría de nuestras observaciones, el comienzo es brusco: la historia de nuestros enfermitos comienza el día antes o data de 2 a 3 días, con temperatura generalmente elevada, cefaleas intensas, rigidez de la nuca y vómitos. Son los síntomas cardinales, que rara vez faltan, acompañados o no de otras manifestaciones como ser: dolores abdominales, artralgias, mialgias, molestias visuales u oculares, constipación, sudoración, petequias. etc.

A veces suelen venir convulsiones y se instala un estado de somnolencia, alternado muchas veces con periodos de agitación psicomotora.

La fiebre no manifiesta ninguna característica especial.

El comienzo en ocasiones suele ser tan brusco que enfermitos vistos en la mañana con un cuadro febril, que se ha interpretado como un estado gripal, con ausencia de todo signo meníngeo, en la tarde han transformado su estado catarral de las vías respiratorias por un franco cuadro meníngeo con embotamiento del sensorio y el cortejo sintomático de una meningitis.

De nuestras 40 observaciones en 29 casos el comienzo es brusco, con temperatura, vómitos, cefaleas, rigidez en la nuca y rápido embotamiento del sensorio.

Al lado de estos cuadros agudos, de co- mienzo brusco, con sintomatología ruidosa, tenemos cuadros solapados, insidiosos con signos clínicos atenuados que es preciso buscarlo pensando en la meningitis. Tenemos varios casos de estos en que sólo algunas manifestaciones nos han hecho sospechar el diagnostico y la punción lumbar nos ha revelado la verdad. Especialmente cierto esto en los lactantes en que el cuadro clínico se presenta en forma atípica con relación al cuadro del niño mayor.

Goppert le da gran importancia a la presencia del meningococo en la sangre considerando a la enfermedad como una sepsis meningocóccica en la cual la meningitis representa la metástasis más frecuente al lado de las oculares laberínticas, endocárdicas y serosas.

Para Lang la meningococcia es una entidad patológica bien definida.

En enfermos que presentan elevaciones térmicas, escalofríos, eritemas, artralgias, mialgias, debe sospecharse meningococcemia, pudiendo establecerse el diagnóstico por hemocultivo o por la curva del oro coloidal, aunque los otros hallazgos del líquido cefalorraquídeo sean negativos.

Agrega, que una pronunciada hipoglucoraquis asociada a signos o síntomas de irritación meníngea es patognomónica de meningitis cerebro espinal, aún cuando no se encuentren meningococos en el líquido o en la sangre, pues suele adelantarse a la aparición de éstos.

Los enfermos por lo general se encuentran en actitud pasiva, posición en gatillo, a veces en excitación psicomotora y muy variable en intensidad.

La rigidez de la nuca es muy manifiesta $\mathrm{y}$ en muchas ocasiones se ha presentado un verdadero opistótono, Los signos de Kernig y Brudzinsky son casi constantes como asimismo la raya meníngea. En los lactantes se presenta siempre la fontanela tensa.

Un signo que casi nunca falta y que hemos constatado con gran frecuencia en nuestros enfermitos es el descrito por Goppert: la exageración del reflejo pupilar al dolor, que se provoca por estímulo de la piel.

A estos signos de gran frecuencia y que son los que conducen al diagnostico, suelen agregarse otras manifestaciones que también las hemos encontrado en nuestros enfermitos. 
Manifestaciones de la piel: púrpura, equimosis o petequias, a veces exantema de intensidad variable.

Herpes labial o del territorio del facial. El herpes suele acompañarse de gingivitis o estomatitis y en ocasiones esto se presenta aisladamente.

En algunas ocasiones suele presentarse abundante sudoración que llama la atención y que constatamos en varias oportunidades.

Las manifestaciones oculares son más raras, nosotros las hemos constatado en pocas oportunidades, se suelen presentar estrabismos, nistagmus, conjuntivitis, iritis.

Las manifestaciones óticas son sólo más raras aún.

De parte de las articulaciones sólo hemos tenido pocos casos y en uno de ellos llegó a la supuración.

Las mialgias suelen presentarse a veces en forma intensa y otras son fugaces.

La punción lumbar es la que da la comprobación diagnóstica.

El aspecto del líquido céfalo raquídeo es variable, puede ser opalescente y ligeramente turbio, hasta purulento franco, a veces dejando salir grumos. En algunas ocasiones se presenta xantocrómico.

La albumina por lo general está muy aumentada, en otras ocasiones el aumento es mayor, pero siempre se encuentra sobre lo normal.

La cantidad de glucosa es variable, más frecuentemente esta baja y aún llega a desaparecer, pero suele estar normal o aumentada. Los cloruros pueden estar normal, aumentar o disminuir.

Las reacciones de albuminas, Pandy, Nonne, Appelt y Weichbrodt son fuertemente positivas.

Generalmente se encuentran una gran cantidad de leucocitos con predominio enorme de los polinucleares.

Al examen directo suele revelarse el Diplococus Gram negativos intra o extracelulares que en nuestros enfermitos se han encontrado 25 veces.

El cultivo que se ha hecho en medios especiales se ha revelado positivo para el meningococo 14 veces.

La velocidad de sedimentación la encon- tramos generalmente aumentada, variando en límites que fluctúan entre $20 \mathrm{~mm}$ hasta más de $100 \mathrm{~mm}$ en la hora, sin que haya ninguna relación con la gravedad del caso. Posteriormente con el tratamiento y la mejoría del estado general, esta baja; pero hemos visto en algunos casos que a los 7 a 10 días, a veces aumenta.

El examen de sangre tampoco revela nada específico, sólo se encuentra una leucocitosis con predominio de los neutrófilos.

\section{Frecuencia}

Según el sexo, en nuestras observaciones encontramos un ligero predominio por el sexo femenino, presentándose 22 de sexo femenino y 18 del sexo masculino.

Con respecto a la edad encontramos una mayor frecuencia alrededor de los 3 a 6 años.

Según las edades tenemos: de

$\begin{array}{lr}1 \text { a } 6 \text { meses............ } & 2 \text { casos } \\ 6 \text { a } 12 \text { meses ......... } & 3 \text { casos } \\ 1 \text { año a } 3 \text { años......... } & 4 \text { casos } \\ 3 \text { años a } 6 \text { años........ } & 14 \text { casos } \\ 6 \text { años a } 10 \text { años...... } & 12 \text { casos } \\ 10 \text { años a } 14 \text { años..... } & 5 \text { casos }\end{array}$

\section{Diagnóstico}

El diagnóstico de los casos confirmados no es difícil sobre todo en tiempo de epidemia. La comprobación se efectúa por el examen del líquido céfalo raquídeo.

La administración temprana de derivados de sulfanilamida perturba el diagnóstico por la rapidez con que regresan los síntomas.

El diagnostico diferencial debe hacerse con el meningismo del tifus abdominal, neumonía, meningitis secundaria a afecciones (del oído, fosas nasales, cara); tifus petequial, la meningitis tuberculosa, sifilítica, etc.

\section{Evolución}

Jackman distingue 4 tipos evolutivos en la enfermedad:

1. Los casos de evolución aguda.

a) Meningitis fulminante.

b) Los casos que evolucionan en 4 a 6 días.

2. Casos de evolución lenta.

a) Con supuración continuada y casi siempre curso intermitente.

b) Con formación de una hidrocefalia interna. 
3. Meningitis del lactante.

4. Casos sumamente leves y abortivos.

Esta división un tanto esquemática no ha perdido hoy día su valor con el tratamiento por los sulfamidos.

Tenemos entre nuestros fallecidos un caso que lo podernos catalogar como meningitis fulminante, cuya enfermedad desde los primeros síntomas, duro alrededor de 15 horas y que falleció en el servicio a las 2 horas de ingresar, como ya lo hemos visto, otra chica de 3 años ingresó con un cuadro meníngeo grave, de comienzo brusco, y a pesar del intenso tratamiento efectuado desde un comienzo, falleció al cuarto día de su ingreso.

Por otra parte, la meningitis del lactante que tiene características especiales en su comienzo y evolución, puede perfectamente hacerse una división especial.

Generalmente en el lactante el comienzo es insidioso, sin presentar el cuadro alarmante del niño mayor.

Corrientemente comienza con un cuadro gripal, con un estado catarral de las vías respiratorias, después se instala un estado de irritabilidad, inquietud alternada con somnolencia, temperatura y vómitos.

Al examen puede observarse la fontanela tensa, rigidez de la nuca. Estas manifestaciones hacen sospechar la meningitis y el diagnostico lo da la punción lumbar.

La evolución clínica de nuestros enfermos se puede casi esquematizar en lo siguiente:

A las 24 horas, por lo general de iniciado el tratamiento, hay caída brusca de la temperatura; enfermos que llegan con 39 y 40 grados, al día siguiente presentan 37 y 37,5 grados de temperatura, siguen 2, 3 ó 4 días con temperatura subfebriles para desaparecer después completamente.

El sensorio que está completamente embotado en casi todos ellos, a las 24 ó 48 horas comienza a despejarse y al tercero o cuarto día están por lo general completamente lúcidos.

Los signos meníngeos son los más tardíos en desaparecer $y$ por lo general la rigidez a la nuca y la raya meníngea, los que persisten por más tiempo. En todo caso, al término de la primera semana o al comienzo de la segunda, no se aprecia ninguna manifestación meníngea.

Es interesante hacer notar que enfermos ingresados en estado gravísimo, al día siguiente se les ha encontrado sentados en la cama pidiendo que comer o pidiendo una revista para leer.

Naturalmente, que estos han sido casos excepcionales: pero por lo general, a las 48 ó 76 horas es corriente encontrarlos en la visita en esta actitud a pesar de tener aún rigidez de la nuca y aun el Kerning y el Brudzinsky positivos.

\section{Complicaciones}

Las complicaciones que hemos tenido en nuestros enfermitos han sido relativamente raras y la mayoría de ellas sin mayores trascendencias.

En uno de los casos, Alejandro F., a los 7 días se queja de dolores en la articulación radiocarpiana derecha, la que se presenta tumefacta y dolorosa. Al día siguiente aparece tumefacto y doloroso el codo y la rodilla derecha. La articulación de la rodilla llega a la supuración y en el pus se encuentran Diplococus Gram negativos. Se inyecta albucid y se repite la punción y la inyección dos días después, mejorando completamente sin dejar anquilosis.

Berta S. niñita de 4 años, ingresa a los 15 días de enfermedad, sin signos meníngeos y con gran excitación psicomotora, disminución de la agudeza visual que llega casi hasta la amaurosis y fotofobia intensa. El examen del líquido céfalo raquídeo comprobó una meningitis meningocóccica.

El especialista diagnosticó una Iritis endógena, la mejoría fue muy lenta, pero quedó en buenas condiciones.

Una complicación grave que se nos presentó fue el de Elba M., de 9 años, que al tercer día, al despejarse el sensorio, pudimos constatar que tenía una sordera absoluta. Consultado al especialista, nos confirmó que los tímpanos estaban sanos y no encontraba nada anormal en el oído externo y medio, interpretando su sordera como una lesión del nervio acústico de carácter irreparable; 15 días después ha continuado igual.

Otras complicaciones de menor impor- 
tancia han sido cefaleas, vómitos, dolores de oído, dolores articulares y musculares fugaces que no han tenido mayor trascendencia. Estas complicaciones podrían atribuirse a intolerancia por la droga, pero algunas veces se han presentado después de suspendida ésta.

\section{Anatomía patológica}

Nuestra experiencia en este sentido es muy escasa, pues sólo tenemos 4 casos que fallecieron en el servicio, con lo cual no se pueden sacar conclusiones definitivas.

En todo caso lo que se encuentra en la anatomía patológica es una congestión intensa de los meninges y del encéfalo con gran aumento de peso del encéfalo, distensión de los ventrículos por hidrocefalia y a veces destrucción, leptomeningitis y placas de fibrina y exudados fibrino purulentos especialmente en la base. En uno de los casos se encontró un punteado fino de color rojo vivo en la mucosa gástrica intestinal. Por lo general, hay cianosis de los órganos visuales.

\section{Tratamiento}

El tratamiento efectuado por nosotros ha sido con Dagenan, Cibazol, Aseptil y un caso con Albucid, ayudado con Completina fuerte o sea Vitamina $C$, inyectable a la dosis 0,10 gr 1 ó 2 veces al día según el caso. Usamos la Completina por considerar la vitamina $\mathrm{C}$ un gran coadyuvante como anti-infeccioso, antitoxico y por hacer más tolerables los preparados sulfamidados como ha sido demostrado y lo dijimos en un trabajo anterior.

El suero no lo hemos usado.

En los enfermos muy graves hemos recurrido a las transfusiones de sangre y esto no ha pasado de 3 casos.

Rara vez hemos tenido necesidad de recurrir a los tónicos cardiacos u otra terapéutica coadyuvante.

Los preparados sulfamidados los hemos usado a la dosis a 15 a 20 centigramos por kilogramo de peso en las 24 horas, iniciando por lo general el tratamiento con una dosis alta, 10 2 gramos según la gravedad y el peso del niño. Rara vez hemos tenido que recurrir a dosis mayores, pero hemos llegado a dar hasta 50 centigramos por kilo en las 24 horas.
La dosis alta la mantenemos hasta que pasen los signos meníngeos completamente, baje la temperatura y la punción lumbar de control nos da el líquido céfalo raquídeo normal.

Después de este control bajamos la dosis paulatinamente manteniéndola algunos días hasta suspenderlo totalmente alrededor de los 12 ó 15 días de iniciada la enfermedad. De los 40 casos tratados, 16 han sido sulfopiridina (Dagenan), 17 con sulfanilamida (Aseptil), 6 con sulfanilamidatiazol (Cibazol) y 1 caso, contra nuestro modo de pensar, se indico Albucid en lavados intrarraquídeos e inyecciones intravenosas, con un total de 3 gramos diarios con resultado fatal.

Los resultados terapéuticos han sido sensiblemente iguales con las tres drogas (Dagenan, Cibazol, Aseptil), a pesar de lo que se ha hecho de los sulfatiazoles que atraviesan con dificultad la barrera meníngea.

Los tres preparados han sido bien tolerados a dosis altas y mantenido el tratamiento por varios días y las intolerancias que se han presentado han sido tan insignificantes que no ha sido motivo para interrumpir el tratamiento.

Los resultados de los tratamientos efectuados ya lo hemos expuesto, hemos presentado las complicaciones y sólo nos queda decir que todos los dados de alta se han ido en perfectas condiciones, sin presentar secuelas orgánicas ni psíquicas (excepción de una sordera que aún permanece en el servicio) y después de su estadía en el hospital van a convalecer al Preventorio de la Cruz Roja de El Belloto que por gestión de la Jefatura Provincial de Sanidad se puso a disposición de estos enfermitos en pabellón aparte y allí se les tiene en observación por 15 días antes de reintegrarlos a sus hogares o a la institución de donde salieron enfermos a fin de evitar todo peligro de contagio para la colectividad.

Presentamos aquí los hechos como los hemos observado, exponemos los resultados obtenidos con nuestro tratamiento y confesamos también los errores cometidos. Sin embargo, nuestro trabajo no es completo, por cuanto no están todos los casos de meningitis que se han presentado en Valparaíso por estar comprendidos sólo los atendidos en la sección niños del Hospital Deformes, cosa que lo ha hecho el Dr. 
Tondreau y por otra parte hasta este momento siguen presentándose nuevos casos. Por este motivo, una vez pasado este brote agudo podemos analizar nuevamente los hechos ya en forma más completa.

\section{Comentario del artículo publicado por los Drs. Pumarino y Grossi}

Corría el 31 de Mayo de1941, cuando "llegaba a la sección Pediatría del Hospital Deformes un niño de 3 años, asilado en la Casa de Huérfanos La Providencia de Valparaíso, con un cuadro clínico que en un comienzo nos desorientó", el pequeño presentaba un cuadro febril asociado a vómitos, cefalea, dolor abdominal, compromiso de conciencia y rigidez de nuca; fue el resultado de la punción lumbar obtenido al día siguiente lo que "nos puso sobre la pista"... "Tres días después ingresaba otro niño del mismo asilo con un carácter análogo $y$ el resultado del examen, el líquido y el cultivo positivo para el meningococo nos daban razón para pensar que estábamos en presencia de casos de meningitis meningocóccica." Con esas palabras relatan los Drs. Pumarino y Grossi el inicio de la epidemia de enfermedad meningocócica en nuestro país ${ }^{1}$.

En aquellos años se consideraba que esta enfermedad afectaba a los niños más grandes, probablemente porque en los más pequeños no se alcanzaba a establecer un diagnóstico previo a un desenlace seguramente fatal; era un hecho universalmente aceptado que "las enfermedades infecto-contagiosas, con excepción de algunas infecciones estreptocócicas, de la tos convulsiva y unas pocas más respetan a los niños menores de un año"3. En la epidemia de los 40, quizás debido a que los médicos estaban más alerta a la sintomatología, se reportó que un cerca de un $10 \%$ de los afectados correspondía a lactantes, grupo en que la tasa de letalidad alcanzaba al $50 \%{ }^{2}$.

A menos de una década de su descubrimiento, fueron publicadas en nuestra revista las primeras experiencias exitosas con las sulfas en el manejo de la meningitis meningocócica. Distinta era la historia de los contagiados de meningingitis neumocócicas en que la mortalidad, pese al tratamiento antibiótico, era mayor al 50\%, y alcanzaba el $100 \%$ de las meningitis a neumobacilos y grippales ${ }^{7}$. Previo a la implementación de la antibioterapia, las meningitis meningocócicas eran tratadas con suero específico, tratamiento con el cual "se obtenía la mejoría de más o menos la mitad de los casos"8. Desgraciadamente, al poco tiempo de su empleo, aparecieron los primeros indicios de resistencia a las sulfas, siendo reemplazada por la recién descubierta penicilina y posteriormente por nuevas generaciones de antibióticos.

Una extraordinaria medida implementada por el Servicio Salud Pública, destinada a acortar los tiempos de inicio de la antibioterapia, factor determinante del pronóstico de la enfermedad, además de combatir "la epidemia de neurosis", fue comenzar tratamiento a los "pies de la cama del paciente" frente a la menor sospecha de enfermedad; para ello se facilitó a los médicos un auto y un maletín equipado con sulfas, jeringas, agujas estériles y drogas cardiovasculares para tratar los casos de colapso. Según relato del Dr. Prizzi, con estas medidas se logró disminuir la mortalidad de $23 \%$ a comienzos de la epidemia a $14,5 \%$ apenas 1 año después ${ }^{2}$. Curiosamente esta tasa de letalidad se ha mantenido relativamente estable en el tiempo, seguramente debido a un subdiagnóstico, en aquellos años, de los casos más severos.

En una época en que las inmunizaciones contra meningococo estaban lejos aún de ser una realidad, eran otras las medidas profilácticas que se fueron implementando, las medidas que se han mantenido con pequeñas modificaciones, en la medida que ha ido mejorando el nivel de vida de la población. Entre dichas medidas destaca la obligatoriedad en la notificación y aislamiento de los enfermos; "tan pronto como el clínico haya formulado un diagnostico de probabilidad se recomienda aislamiento riguroso, además de prohibirse las visitas, que los cuidadores llevaran mascarilla y delantal, las secreciones y excreciones serán recibidas en un recipiente que se pueda incinerar o, en su defecto, en una solución desinfectante, formol al 10\% o ácido fénico al 2,1\%... además, precede evitar las aglomeraciones, los esfuerzos fisicos e intelectuales exagerados, la expo- 
sición prolongada al sol, los enfriamientos y todo lo que pueda disminuir las resistencias" "3. Como medida adicional se implementó la administración de sulfonilamida durante 14 días a los contactos.

Difícil es saber cuál de todas las medidas descritas tuvo el mayor impacto en el control de la epidemia, si fue el tratamiento a los pies de la cama del paciente, o bien el aislamiento y profilaxis a los contactos; podría deberse en parte a la efectividad de la antibioterapia o quizás la mejoría del nivel de vida de nuestra población. Lo más probable es que debamos agradecer a la sumatoria de cada una de las medidas de Salud Pública, implementadas gracias al profesionalismo e integridad de muchos maestros de nuestra historia de la medicina.

\section{Referencias}

1.- Pumarino H, Grossi V: Aspecto clínico de los casos de meningitis meningocóccica que se presentaron en
Valparaíso de 21 de mayo al 12 de septiembre de 1941 en la sección de niños del Hospital Deformes. Rev Chil Pediatr 1942; 13 (1): 3-20.

2.- Prizzi M: A Severe Epidemic of Meningococcus Meningitis in Chile, 1941-1942. American Journal Of Public Health 1944: 231-8.

3.- Romero H: Anotaciones sobre meningitis meningocócica. Rev Chil Pediatr 1943; 14 (1): 14-7.

4.- Meneghello J, Schwarz J, Steeger A: Síndrome de Waterhouse-Friderichsen. Rev Chil Pediatr 1944; 15 (12): 1010-23.

5.- Wilhelm J, Villena $R$ : Historia y Epidemiología del Meningococo. Rev Chil Pediatr 2012; 83 (5): 533-9.

6.- Selbst $S$ : Malapraxis en la emergencia pediátrica: Error en el diagnóstico de meningitis y eningococemia/bacteriemia. Arch Pediatr Urug 2001; 72 (suppl. 1): S26-S33.

7.- Del Carril M, Foley G, Larguia A, Sojo E: La sulfamidoterapia en el lactante. Rev Chil Pediatr 1942; 13 (8) 701-14.

8.- Llodra G, Volosky R, Costa A: Meningitis purulentas en el niño: Experiencia sabre 61 casos tratados con Sulfas y Antibióticos. Rev Chil Pediatr 1953; 24 (10): 369-79. 